\title{
LA BÚSQUEDA DE UN TIEMPO PERDIDO, EN LA PELÍCULA LOVE LETTER (CARTA DE AMOR) DEL DIRECTOR SHUNJI IWAI
}

\section{ORLANDO BETANCOR}

Universidad De La Laguna

Resumen: Este artículo aborda el tema de la búsqueda de un tiempo perdido, imposible de volver, en la película Love Letter (Carta de amor, 1995), dirigida por el realizador japonés Shunji Iwai. Esta película narra la historia de una joven que escribe una carta a su novio, fallecido en un trágico accidente hace dos años, y sorprendentemente recibe una respuesta. Igualmente, esta cinta trata aspectos como el amor, el dolor y los designios del destino.

Palabras clave: Cine japonés, recuerdo, amor, dolor, destino
Abstract: This article approaches the subject of the search of a lost time, impossible to return, into the film Love Letter (1995), directed by the Japanese filmmaker Shunji Iwai. This movie tells the story of a young woman who writes a letter to her boyfriend, expired in a tragic accident two years ago, and surprisingly she receives a response. Likewise this film deals with aspects such as love, pain and the designs of the destiny.

Keywords: Japanese cinema, memory, love, pain, destiny

Résumé: Cet article aborde le sujet de la recherche du temps perdu, impossible de revenir, dans le long métrage Love Letter (1995), dirigé par le réalisateur japonais Shunji Iwai. Ce film raconte I'histoire d'une jeune femme qui écrit une lettre à son fiancé, décédé dans un tragique accident il y a deux ans, et d'une facon surprenante reçoit une réponse. Également, ce long métrage traite des aspects comme l'amour, la douleur et les desseins du destin.

Mots-clés: Cinéma japonais, le souvenir, l'amour, la douleur, le destin
The search of a lost time, into the film Love Letter of the filmmaker Shunji Iwai

La recherche du temps perdu, dans le long métrage Love Letter du réalisateur Shunji Iwai
BIBLID [(2015), 5; 147-156]

Recep.: 03/06/2014 Acept.: 20/03/2014 


\section{Introducción}

Este ensayo pretende abordar el tema de la búsqueda de un tiempo perdido, que es imposible de recuperar, en la película Love Letter (Carta de amor, 1995), dirigida por el director japonés Shunji Iwai ${ }^{1}$. El guión de esta cinta es también obra de este realizador y su deslumbrante fotografía es creación del desaparecido Noboru Shinoda, habitual en las producciones de este cineasta. Este drama románico está protagonizado por Miho Nakayama, que interpreta un doble papel, Etsushi Toyokawa, Bunjaku Han, Takashi Kashiwabara y Katsuyuki Shinohara, entre otros actores. Este largometraje muestra la realidad actual de una muchacha llamada Hiroko que perdió a su prometido en un accidente de escalada hace dos años. Esta joven no ha superado todavía su pérdida y está sumida en una profunda tristeza. Guiada por la desesperanza y la soledad, la muchacha decide enviar una carta a su antiguo novio, esperando una señal de los astros, a la dirección en la que éste residía en su ciudad natal. Asombrosamente, al cabo de poco tiempo, recibe una respuesta firmada por Itsuki, el nombre de su pareja. La misiva la ha escrito una mujer que se llama igual que él, una compañera de sus años escolares. La sorpresa inicial de Hiroko se transforma en una inquietante correspondencia entre ambas féminas, cuyas vidas se interrelacionan a través de misteriosos vasos comunicantes. A lo largo de este singular recorrido epistolar, su protagonista intentará romper con las cadenas que la atan al pasado, acabar con la amargura que asola su alma y mirar hacia el futuro con esperanza. Entre los temas que trata esta cinta se encuentran, entre otros, los designios del destino, la muerte y el amor.
A través de este análisis fílmico, se ha intentado realizar una aproximación a la personal estética visual de este innovador cineasta japonés en la primera de sus creaciones para la gran pantalla. Además, este artículo se centra especialmente en su universo simbólico que toma forma, en esta película, a través de un viaje por un tiempo perdido, un recorrido por los recuerdos del ayer, que transforma la existencia de dos mujeres. Asimismo, para la redacción de este ensayo, se han utilizado diferentes estudios sobre las últimas tendencias en el cine del país del Sol Naciente, los cuales nos han permitido profundizar en diversos aspectos de la labor creadora de este joven realizador cinematográfico.

\section{Los laberintos de la memoria}

Este film comienza con el deslumbrante plano de la nieve cayendo sobre el hermoso rostro de Hiroko Watanabe, que yace inmóvil, sobre el níveo lecho de una montaña, situada al este de la prefectura de Kobe, en Japón. Poco después, la muchacha se levanta del blanco sudario que la envuelve y asiste al oficio religioso en memoria de su novio, Itsuki Fujii, fallecido dos años atrás en un trágico accidente. Una vez concluida la ceremonia, la madre de su prometido le invita a entrar en su casa y le enseña el anuario del instituto donde estudió su hijo y le comenta que anteriormente la familia vivía en Otaru, en la isla de Hokkaido, en la parte norte del archipiélago japonés. También, le comenta que su antigua residencia hace ya tiempo que fue demolida para construir una autovía y que su vástago cambió

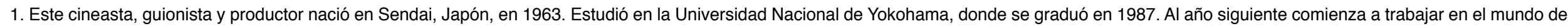

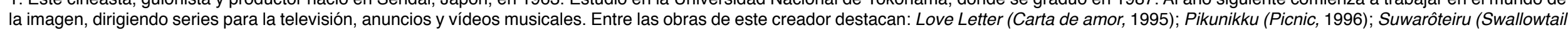

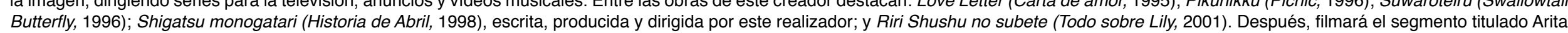

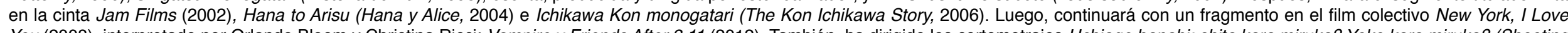

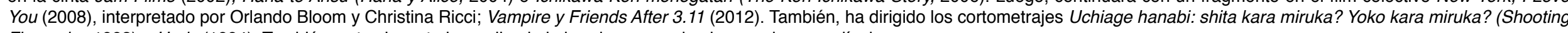
Fireworks, 1993) y Undo (1994). También, este cineasta ha realizado la banda sonora de algunas de sus películas. 
de centro educativo antes de terminar el último año de la secundaria. Movida por un deseo irrefrenable, la joven busca la antigua dirección de su prometido, que aparece en la publicación, y la escribe en tinta en su antebrazo, transformándose en un singular tatuaje impreso en su piel que refleja su profundo dolor. Sorprendentemente, poco tiempo después, recibe una respuesta, firmada por Itsuki Fujii. Por azares del destino, la carta es entregada a una muchacha, que trabaja en la biblioteca pública de Otaru, la cual posee el mismo nombre que su novio y estuvo con él en su misma clase en el instituto. La joven, que tiene un fuerte resfriado, se muestra desconcertada al leer esta misiva y no consigue recordar a su enigmática remitente. Se da la extraordinaria casualidad de que estas dos mujeres son físicamente idénticas, como dos gotas de agua, pero sus personalidades son completamente diferentes: Hiroko es introvertida y sosegada, mientras que Itsuki es inquieta y desenvuelta.

Al recibir este escrito, Hiroko va en busca del mejor amigo de su novio, Akiba Shigeru, un joven analítico y voluntarioso, que también estuvo presente en el accidente que le costó la vida a su compañero. Ella está llena de ilusión por la llegada de esta misiva, que cree de origen celestial, lo que demuestra que no ha logrado sobreponerse todavía a la muerte de su pareja. Por otro lado, en el alma del chico se alberga un profundo dolor, pues está enamorado de esta joven. Él desea liberarla de los lazos que la aprisionan en el ayer y acabar con la presencia invisible, la imagen de su prometido, que se interpone entre ellos. En su taller de fundición, este artista del vidrio intenta besarla, pero ella se muestra poco receptiva ante esta muestra de afecto. Luego, este personaje, intrigado por este misterio, se toma la licencia de escribir a Itsuki Fujii y le pide una prueba de su existencia, un documento que demuestre su identidad. Después, Hiroko recibe otra carta con una copia del carné de conducir de la bibliotecaria y el chico se queda sorprendido al comprobar que es una mujer la que posee el mismo nombre y apellido que su amigo. En este nuevo escrito, la chica de Otaru le pide a su remitente que no vuelva a ponerse en contacto con ella nunca más, pues la cree una simple enajenada. En ese momento, mientras Akiba e Hiroko conversan en un bar, éste se queja de la crueldad de la muchacha, al mencionar constantemente a su antiguo novio, y le confiesa que ha estado enamorado de ella desde el primer día que la vio. Además, muestra su deseo de convertirla en su esposa.

Posteriormente, a requerimiento del artista, ambos emprenden un viaje hasta Otaru buscando respuestas a este enigma y poder conocer a la otra Itsuki Fujii. El chico espera que, al encontrarse con la destinataria de las misivas, Hiroko rompa con el pasado y comience una nueva vida junto a él. Mientras tanto, el resfriado de la bibliotecaria empeora y su madre emplea una estratagema para que vaya al hospital. Paralelamente, Akiba y la joven buscan primero en la nueva autovía cualquier rastro de la antigua casa de su novio y, luego, comprueban la auténtica dirección postal a la que ha enviado las cartas, la verdadera vivienda de Itsuki. Como la muchacha no está en su domicilio en ese momento, deciden esperar en el exterior del inmueble, mientras Hiroko le escribe una nota, explicándole la confusión que ha habido con el nombre de su prometido, obviando que éste ya no se encuentra en este mundo. La chica, ante la tardanza de la bibliotecaria, decide abandonar el lugar. Después, un taxista nada más entrar en su vehículo, les dice que acaba de dejar a una pasajera, justo igual que ella, que podía ser su gemela, hace sólo un instante. Al día siguiente, antes de dejar la ciudad, las dos mujeres coinciden casualmente en un paso de peatones. Itsuki va en bicicleta y, en ese momento, escucha que una voz femenina pronuncia su nombre. Ésta vuelve la cabeza, pero no logra distinguir a nadie, pues una multitud de personas empieza a rodear a las dos féminas en unos segundos. Cuando regresa a su hogar, Hiroko pide ver nuevamente el anuario del instituto y comprueba que realmente existían dos Itsuki Fujii en el mismo centro educativo y que ella anotó la dirección de la muchacha en vez de la 
de su pareja. Entonces, observa la foto de la compañera de estudios de su novio y advierte el sorprendente parecido existente entre ambas. En ese instante, se despierta en su alma el fantasma de los celos, pues cree que su prometido aún estaba enamorado de esta joven cuando la conoció.

A partir de ese momento, Hiroko le pide a la otra Itsuki que comparta con ella los recuerdos que tiene de su novio. Entonces, aparecen en su mente antiguas imágenes, que se sitúan en la película como continuos flash-backs, que muestran escenas de sus tiempos de colegiales como las constantes bromas de las que eran objeto, desde el primer día de clase, por llamarse exactamente igual y sobre el hipotético amor que pudiera existir entre ambos. En esa época, el joven Itsuki se mostraba como un chico serio, retraído y bastante complejo. Además, en ese momento, se les encarga a los dos estudiantes colaborar en las labores bibliotecarias del centro, aunque ella tenía que ocuparse de todas las tareas y él siempre se comportaba de una manera distante, leyendo en algún rincón. En aquel tiempo, el muchacho inventa un curioso y elaborado juego: sacar en préstamo de la biblioteca libros, que nadie anteriormente había leído, para que su compañera inscribiera su nombre en las tarjetas de lectura de los mismos. Después, Hiroko le envía a la chica de Otaru una cámara polaroid para que tome instantáneas de los lugares que su novio frecuentaba en esa época: la cancha de deportes, donde correría su última carrera, y las aulas del instituto. Cuando la chica entra en el interior del centro, se tropieza con una antigua profesora que la invita a visitar las instalaciones de la biblioteca. Allí, las alumnas, que colaboran en estas dependencias, se ríen nerviosas cuando le presentan a la joven, pues han elevado su figura a la categoría de "leyenda" por su gran afición a la lectura. Éstas han creado un curioso entretenimiento que consiste en buscar los libros que Itsuki Fujii había retirado en préstamo durante esta época y hacer una lista con los mismos. Entonces, ella les explica que, en realidad, es el nombre de su compañero el que aparece en las fichas de los volúmenes y no el suyo. Antes de salir del centro, la maestra le revela que el muchacho había muerto en un accidente dos años atrás.

Mientras tanto, Akiba quiere llevar a Hiroko a la montaña, donde su novio perdió la vida, para que ésta se enfrente de una vez con los fantasmas que asolan su mente. Se dirigen en tren hasta este lugar y pasan la noche, en la cabaña de Gaji, otro de los amigos de su prometido que también fue testigo de su muerte. Los tres comparten, durante la cena, diferentes momentos vividos con el desaparecido. En ese instante, Hiroko dice lo siguiente: "A mí me dejó muy buenos recuerdos... Pero yo siempre quería más y más. Le perseguí hasta después de muerto, suplicando por un poco más. No soy más que una egoísta". Por su parte, la otra Itsuki también alberga una pérdida en su alma, pues su padre murió de neumonía hace diez años. La muchacha empeora de su resfriado y cae desmayada por la fiebre en la cocina de su casa, cumpliendo el mismo sino que su progenitor. Su abuelo llama inmediatamente a una ambulancia, pero ésta tarda una hora en llegar, pues se encuentran en medio de una fuerte tormenta de nieve. El anciano, ante la demora de los equipos médicos, decide llevar a su nieta sobre su espalda hasta el hospital. En la mente de este hombre, se encuentra presente la muerte de su propio hijo, al que tuvo que portar durante cuarenta minutos en similares circunstancias, pero éste finalmente fallecería. Al llegar al centro hospitalario, tanto la joven como el anciano son ingresados a toda prisa en el servicio de urgencias. Poco después, en la cabaña de la montaña, Akiba despierta a Hiroko para que vea el amanecer junto a él. Señala la cima de un monte, a lo lejos, y le dice que el espíritu de su camarada descansa en aquel lugar. Luego, éste insiste para que la joven se acerque, lo más cerca que pueda, a la estampa de este cerro nevado. En este momento, la muchacha empieza a invocar en voz alta el alma del desaparecido, repitiendo las mismas palabras que le escribió en la primera carta al más allá, una y otra vez, mientras rompe a llorar desconsoladamente. De esta manera, empieza 
su particular catarsis interior que le permitirá acabar con esta etapa de su existencia y comenzar otra completamente diferente. Paralelamente, en el hospital, a miles de kilómetros de distancia, la bibliotecaria se despierta y murmura los mismos términos que Hiroko está pronunciando, en ese preciso momento, como una dolorosa letanía. En ese instante, una furtiva lágrima surca su rostro. Más tarde, ésta recuerda un acontecimiento perdido en su memoria. Durante el período del luto, dedicado a su padre, la muchacha recibe la visita en su casa del joven Fujii. Éste le pide que devuelva el libro, À la recherche du temps perdu, del escritor Marcel Proust, que había pedido en préstamo a la biblioteca y le dice que no puede entregarlo por sí mismo. Cuando la chica vuelve al instituto una semana después, el joven había cambiado de centro escolar sin despedirse de nadie. Ésta sería la última vez que le vería.

\section{Universo temático}

En esta original cinta, llena de referentes simbólicos, encontramos en primer lugar el tema de la búsqueda de un tiempo perdido, factor determinante en este relato cinematográfico. Así, en este recorrido existencial, vislumbramos a Hiroko, una joven que vive obsesionada con la imagen del hombre que una vez amó y cuya presencia no ha podido borrar de su mente. Ésta intenta desesperadamente, impulsada por un intenso sentimiento de nostalgia, conservar intacta su memoria. Cuando entra en contacto con la otra Itsuki, hace todo lo posible para que ésta rastree en su pasado los recuerdos de juventud de su pareja y se los transmita con detenimiento. De esta manera, a través de la lectura de sus cartas, este personaje los puede recrear en su pensamiento y sentirlos como si los hubiera vivido en su compañía. En segundo lugar, encontramos al veleidoso destino que con sus inescrutables designios establece inquietantes vínculos de unión entre Hiroko e Itsuki, esta última convertida en su otro yo, su rival inconsciente en el corazón de su prometido. Junto a los caprichos del azar, encontramos la presencia de la muerte, cuya imagen simbólica se percibe en la visión de la nieve que cae sobre el paisaje japonés con su hipnótica belleza. Su aura premonitoria se vislumbra, desde el comienzo de la película, cuando Hiroko entra en la habitación de su prometido y contempla el cuadro de una montaña nevada, que estaba pintando el joven poco antes de su fatal desenlace, y que es el mismo lugar donde perdería posteriormente la vida. Asimismo, el poder de evocación del frío invierno, como alegoría del sueño eterno, se percibe en la secuencia del recuerdo de la pequeña Itsuki que contempla en el hielo la imagen congelada de una libélula, mientras le anuncian, en ese momento, el fallecimiento de su progenitor. También, la simbología del inexorable fin de la existencia humana, asociada con la efímera floración de los cerezos, se observa en el diálogo sostenido entre una compañera de trabajo e Itsuki, cuando éstas mencionan el título de la poética narración corta de Motojirô Kajii, Bajo los cerezos (Sakura no ki no shita ni wa, 1928), y un fragmento de la obra del novelista y ensayista Ango Sakaguchi, En el bosque, bajo los cerezos en flor (Sakura no mori no mankai no shita, 1947). Además, el vacío de la ausencia, por la pérdida de los seres queridos, toma forma en esta cinta en la figura del novio de Hiroko y en la del padre de Itsuki, desaparecido a temprana edad.

Igualmente, el amor, como eje fundamental de la obra de este cineasta, domina en toda su extensión en esta película. De esta forma, el singular juego que emplea el joven Itsuki Fujii, a través del préstamo de libros, refleja, en palabras de una de las estudiantes de la biblioteca escolar, que el muchacho estaba profundamente enamorado de su compañera de estudios, pues este pasatiempo tenía por objeto únicamente captar su interés. Frente al sentimiento afectivo, se encuentra el desamor que se atisba en las figuras de los padres del novio de Hiroko, cada día más distanciados el uno del otro. 


\section{Singular estética visual}

Representante del nuevo cine japonés, que surge en la década de los 90 , Shunji Iwai se ve a sí mismo como un eizo sakka ${ }^{2}$ o artista visual. Este realizador, a través de su atrayente estilo fílmico y su montaje ágil, ha sabido captar los gustos del público de su época que ha crecido con la televisión. De esta manera, intenta acercarse a los gustos de las nuevas generaciones de telespectadores y sus historias reflejan el tipo de vida que éstos llevan en la actualidad ${ }^{3}$. Además, su estética posee muchos puntos de contacto con la cultura pop japonesa de los últimos tiempos, lo que le han convertido en uno de los más importantes cineastas jóvenes del país del Sol Naciente. Sobre estos últimos aspectos, el crítico Ángel Sala comenta lo siguiente: "Fue a partir de Love Letter cuando Iwai conectó con un público autóctono que capta tanto el lenguaje como el mensaje del director y que le ha seguido en sus siguientes películas como: Swallowtail Butterfly o All about Lily Chou-Chou, que compondrían una obra delimitada por una coyuntura social expresa y un método expositivo que ha desarrollado un propio sistema narrativo y visual. Es muy posible que su cine haya sido, hasta el momento, en exceso deudor de una época y de un paisaje anímico, pero, como ocurrió con muchas obras de la nouvelle vague o el nuevo cine alemán, sus películas son absolutamente modernas a la vez que incorpora elementos del clasicismo, al igual que las primeras obras de Godard o Herzog" ${ }^{4}$. Asimismo, este director otorga un sentido esencial a la imagen, apoyándose en las nuevas tecnologías audiovisuales, e investiga para mostrar al espectador una realidad oculta en medio de situaciones marcadas por el azar y la presencia de personajes frágiles y vulnerables. Además, algunos críticos han recalcado que en sus primeras obras, en consonancia con los demás realizadores de su generación, este autor se centra más en lo privado que en la realidad que le circunda, mientras que a menudo enfatiza el estilo visual sobre la sustancia narrativa ${ }^{5}$. Por su parte, el ensayista Luis Miranda comenta, en las siguientes líneas, algunos rasgos característicos de la producción fílmica de este creador: “(...) La fidelidad a los sentimientos propios como autoafirmación individual frente a la paradoja de la repetición; la capacidad de ensoñación, la insistencia y la tendencia al repliegue íntimo; la ahistoricidad de un relato hecho de atmósferas más que de lugares, y la idoneidad del período juvenil, tiempo de escisiones, para ilustrar la propia condición escindida de toda una sociedad; la casualidad como determinismo del relato y la virtualidad y fluidez de la memoria y la percepción podrían definir, con el ejemplo de las películas de Iwai como faro, algunas claves para una taxonomía del relato amoroso en los años de Internet"6.

La obra, objeto de este estudio, fue el primer largometraje de su director y supuso un enorme éxito de público en Japón el año de su estreno. Así, esta cinta estuvo en cartel durante catorce semanas en las salas de exhibición de Tokio $^{7}$, un lugar reservado sólo para las grandes producciones norteamericanas. Además, recibió una amplía cobertura en los medios de comunicación japoneses y obtuvo también una gran repercusión en otros países asiáticos. De la misma manera, esta película, filmada casi en su integridad en Hokkaido, principalmente en la ciudad de Otaru, fue elogiada por la crítica especializada por su estilizada realización fotográfica y la

2. SCHILLING, M.: Contemporary Japanese film. Boston, Weatherhill, 1999, p. 71.

3. Ibídem, p. 71

4. SALA, A.: "Miradas virtuales sobre un mundo frágil: Shunji Iwai y Sabu", El principio del fin: tendencias y efectivos del novísimo cine japonés. Barcelona, Paidós, 2003 , p. 84.

5. SCHILLING, M., op. cit., p. 36 .

6. MIRANDA, L.: Takeshi Kitano. Madrid, Cátedra, 2006, p. 350

7. SCHILLING, M., op. cit., p. 70 
destacable doble actuación de su actriz protagonista. Igualmente, este largometraje ofreció un aire nuevo, lleno de poderosa energía, a la industria cinematográfica del país del Sol Naciente de aquel momento. Por otra parte, Ángel Sala establece puntos de contacto entre esta cinta y el Shojô-manga, un tipo de cómic japonés, cuyo público prioritario, pero no exclusivo, es joven y femenino. Su temática preferente es la amorosa, aunque también es habitual incluir tramas de acción para hacerlo más accesible a un público más amplio ${ }^{8}$. También, este ensayista ha expresado lo siguiente sobre esta producción: "Para su primer filme estrenado en Japón, Love Letter, Iwai compuso una historia de indudables ecos femeninos, utilizando ciertos clichés reconducidos desde su experiencia televisiva y de videoclip y ensamblados en una película estrictamente cinematográfica, que aparentemente resultaba clásica y evocadora pero que es sorprendentemente radical e innovadora"'. Esta intencionalidad, centrada en una audiencia claramente femenina, queda patente en las siguientes notas de este mismo autor: “(...) el director de Picnic nunca abandonó su objetivo de realizar un filme de mujeres y para mujeres que resolviera con éxito el problema de no ser aceptado por el público masculino. En Love Letter los hombres quedaban en un segundo nivel, pero la poderosa magia de las relaciones entre las dos mujeres protagonistas convirtieron el filme en un poderoso y fascinante objeto de seducción"10. Además, hay que resaltar que el montaje de esta producción cinematográfica es también obra de este polifacético realizador. Así, después de escribir el guión, cada imagen de su storyboard la grabó en el ordenador, hasta que filmó la película entera antes de ponerse delante de la cámara ${ }^{11}$. De igual forma, instantes con una intensa carga dramática, como es la negación del deseo en la escena en la que Akiba intenta besar a Hiroko, los concibe este cineasta a través de planos cortos, extremos ángulos fotográficos y cambios del ritmo de edición. Además, la experiencia de este realizador en la dirección de vídeos musicales y anuncios televisivos, se puede apreciar claramente en la secuencia premonitoria en la que Itsuki se encuentra en la sala de espera de un centro médico, se duerme en un banco y tiene una terrible pesadilla, en la que contempla, en medio de una delirante atmósfera, la imagen espectral de una camilla, donde luego aparece su progenitor en grave estado, rodeado de personal sanitario, lo que presagia su próximo ingreso en este mismo hospital.

Igualmente, Ángel Sala reseña estas líneas sobre esta película y sus diferencias con los filmes del director japonés Sabu, seudónimo de Hiroki Tanaka: "Love Letter es un cine de espejos, que juega con la relatividad de la realidad, que cuestiona continuamente la percepción de los sucesos y la inmovilidad de la memoria. Para Iwai, esta última no es un disco duro hermético que pueda permanecer intacto, sino un peculiar mecanismo abierto a la emoción y la percepción, donde lo casual funciona, de un modo muy diferente que en las películas de Sabu, por interrelación y no por el imprevisto. Iwai, ejerciendo de un Proust moderno, busca el tiempo perdido mediante la relación dialéctica, la magia epistolar, ejerciendo el derecho a reivindicar la relatividad de los recuerdos, el descartar la verdad desnuda de nuestras memorias y buscar el camino de la necesaria distorsión de la poesía en nuestras vidas, de recrear nuestro propio pasado y reconstruir, desde lo soñado, nuestro futuro"12. Asimismo, Love Letter resume la estética

8. MIRANDA, L., op. cit., p. 349

9. SALA, A., op. cit., p. 94

10. Ibídem, p. 96

11. SCHILLING, M., op. cit., p. 71.

12. SALA, A., op. cit., p. 96 
y el poder de seducción de este cineasta y se encuentra en la línea de otras de sus producciones como Historia de Abril (1998) o Hana y Alice (2004). También, la fotografía de estos dos filmes es creación de Noboru Shinoda. En la primera de estas cintas, que conserva la estructura formal de un Shojômanga, su protagonista, Uzuki Nireno, interpretada por la estrella de la canción Takako Matsu, es asidua a una librería donde trabaja, a media jornada, el chico del que está enamorada desde sus años de instituto. Esta joven ha dejado atrás su pueblo natal, en Hokkaido, para ir al mismo centro educativo en la capital japonesa, la universidad de Musashino, donde estudia este muchacho, con la esperanza de encontrarse con él. En este film, destaca la imagen alegórica de la lluvia purificadora, en su escena final, que supone el reencuentro definitivo de la muchacha con su antiguo amor. De igual forma, Ángel Sala establece lo siguiente sobre Love Letter y la cinta anteriormente mencionada: "(...) Si en otras de sus obras se detendrá en el devenir de los acontecimientos colectivos, en estas dos obras fundamentales que son Love Letter y April Story el director estableció los lazos entre tiempo pretérito y presente, entronizando el poder del recuerdo y la capacidad de ensoñación como únicas vías de supervivencia en el intuido y frenético mundo urbano. Son puntos de apoyo que Iwai traza para que a sus personajes no se los lleve la marea uniforme e impersonal que domina el todo, enmarcando situaciones, a través de álbumes colegiales o lluvias redentoras. Es un cine que convierte el instante en motor de la acción, la mirada centrífuga en razón de su narrativa, que busca el tiempo perdido y la mirada ausente a través de la percepción del detalle, en suma, una isla de humanismo posmoderno en clave de misiva mainstream decididamente irresistible"13. Igualmente, su largometraje Hana y Alice, obra estrenada en Internet y cuya partitura original es creación también de este realizador, es una historia romántica definida por la amistad entre dos adolescentes y la obsesión de una de sus protagonistas por un muchacho. También, se pueden encontrar otros referentes comunes en la obra de Shunji Iwai en el segmento, filmado por éste, dentro de la cinta colectiva New York, I Love You (2008), producida por Emmanuel Benbihy y Marina Grasic, y en la que participan distintos realizadores como Jiang Wen, Mira Nair o Shekhar Kapur. Cada una de las once narraciones cortas, que la componen, dura alrededor de diez minutos. En el fragmento de este cineasta, un joven compositor, que ultima una partitura para un film de animación, y la secretaria de su director entablan una relación, la cual se inicia a través del hilo telefónico y del correo electrónico para encontrarse finalmente frente a frente. Este segmento se caracteriza por abordar el tema de la búsqueda del amor en la realidad contemporánea y el empleo de delicados haces de luces que crean suaves claroscuros en el interior de las estancias. También, se han encontrado paralelismos temáticos entre la cinta, objeto de este ensayo, y la obra de otros jóvenes realizadores japoneses como Naomi Kawase, quien ha tratado el tema del vacío de la ausencia y la pérdida de los seres queridos en distintos filmes como Sharasojyu (Shara, 2003) y Mogari no mori (El bosque del luto, 2007); y el cineasta Hirokazu Kore-eda, concretamente con su largometraje Maboroshi no hikari (Maborosi, 1995). Esta última película está protagonizada por Makiko Esumi, Tadanobu Asano y Takashi Naitô, la cual trata sobre la muerte y el significado de la existencia. Asimismo, el argumento de esta cinta también se ha relacionado con varias producciones del director polaco Krzysztof Kieslowski como La double vie de Véronique (La doble vida de Verónica, 1991), que aborda el tema de la doble identidad; Trois couleurs: Bleu (Azul, 1993); y Trois couleurs: Rouge (Rojo, 1993). Además, Ángel Sala establece vínculos de unión entre esta película y otros títulos del realizador español Julio Medem como Los amantes del círculo polar (1998) o Lucía y el sexo (2001). De la misma forma, este ensayista ha encontrado similitudes estilísticas con otros importantes filmes como In the

13. SALA, A., op. cit., p. 97 
Mood for Love (Deseando amar, 2000), del cineasta hongkonés Wong KarWai, y Qianxi manbo (Millennium Mambo, 2001), del director taiwanés Hou Hsiao-Hsien.

Por otra parte, esta cinta supuso el lanzamiento en la gran pantalla de la conocida modelo y cantante japonesa Miho Nakayama que da vida, como se ha comentado anteriormente, a las dos protagonistas. Por su labor en esta producción, consiguió varios premios en diferentes festivales como los Blue Ribbon Awards, el Yokohama Film Festival y el Hochi Film Award. Asimismo, durante estos años, la artista ha compaginado su carrera tanto en el cine, con títulos como Tokyo biyori (1997) y Sayonara Itsuka (2010), como en la televisión. Igualmente, destaca la interpretación de los demás actores secundarios de este largometraje ${ }^{14}$. Además, esta película consiguió más de una decena de galardones en distintos certámenes cinematográficos como el Cleveland International Film Festival, el Montreal Film Festival, el Yokohama Film Festival, y recibió tres premios de la Academia Japonesa.

\section{Conclusiones}

En este recorrido por los entresijos del alma humana, marcado por la nostalgia, el dolor y la ausencia de los seres queridos, la protagonista, tras una particular catarsis emocional, se liberará de los vínculos que la aprisionan en el pasado, desterrará la tristeza de su espíritu y comenzará una nueva etapa de su existencia sin volver la vista atrás. Finalmente, en este film, Hiroko le remite a Itsuki todas las cartas que le ha enviado, a lo largo de este intercambio epistolar, pues considera que sus recuerdos le pertenecen por completo. En esta postrera misiva, la joven le dice que tiene el presentimiento de que el nombre, escrito en las tarjetas de lectura de los libros de la biblioteca, corresponde al suyo y no al de su novio. Poco después, la muchacha recibe en su domicilio la visita inesperada de las chicas del instituto. Éstas le muestran el último volumen que el joven Fujii le dejó en su casa y ella entregó en su nombre. En el reverso de la ficha de préstamo de este ejemplar, las alumnas encontraron un retrato a lápiz de la muchacha realizado por su compañero de estudios y que ella nunca había visto hasta ese instante. Ella, profundamente conmovida, muestra su sorpresa ante las alumnas y no sabe si guardar la tarjeta en su bolsillo o colocarla en el interior del libro. Comprende en ese momento que el muchacho siempre estuvo enamorado de ella y que detrás de su fría apariencia se encontraba un alma sensible que jamás le declaró sus intenciones abiertamente. En lugar de expresarle por escrito a Hiroko este acontecimiento, esta cinta concluye con la imagen de Itsuki que dice mentalmente las siguientes palabras: "Querida Hiroko, me siento avergonzada. Así que no voy a mandarte esta carta".

Shunji Iwai ha creado en esta producción un sutil universo visual que se caracteriza por sus elaboradas atmósferas, llenas de sensibilidad y armonía. Igualmente, su personal estética se puede observar con claridad en este film, entre otros ejemplos, en la vaporosa imagen de unas cortinas que el viento mueve en el interior de una pequeña biblioteca; en los pausados movimientos de una joven que desciende una colina en medio de un níveo paisaje invernal; $y$ en la plástica visión de la nieve que cae suavemente sobre las ramas de los árboles de un jardín detrás de los cristales de una ventana. Asimismo, sobresalen los juegos de luces y sombras que se perciben en el

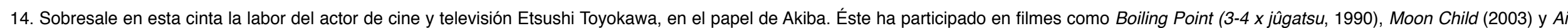

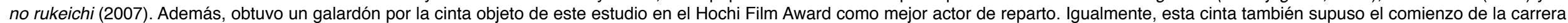

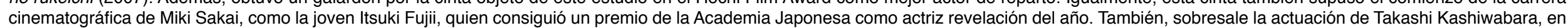
cinematografica de Miki Sakai, como la joven
el papel del joven novio de la protagonista. 
interior de veladas estancias, cargadas de magia, en un depurado equilibrio compositivo. También, destaca en este largometraje la banda sonora -repleta de intimismo y poesía-, compuesta por Remedios, que resalta los momentos de mayor intensidad dramática del mismo. Además, sobresale la habilidad de este cineasta al mostrar en esta obra la complejidad psicológica de unos personajes, dominados por profundos dilemas interiores, que buscan respuestas a sus interrogantes existenciales.

En esta delicada cinta, exquisitamente filmada, su realizador nos ha mostrado el tránsito por los abismos del dolor de una joven que, siguiendo los designios del destino, encontrará a una mujer, físicamente igual a ella, quien le ayudará en este singular peregrinaje por los vestigios del ayer. Igualmente, estas dos féminas recorrerán el espacio de la memoria en busca de un tiempo perdido que sólo existe en su recuerdo. 